\title{
Quantum Computation by Geometrical Means
}

\author{
Jiannis Pachos
}

\begin{abstract}
A geometrical approach to quantum computation is presented, where a non-abelian connection is introduced in order to rewrite the evolution operator of an energy degenerate system as a holonomic unitary. For a simple geometrical model we present an explicit construction of a universal set of gates, represented by holonomies acting on degenerate states.
\end{abstract}

\section{Prologue}

Abelian Sha and non-abelian Wil geometrical phases in quantum theory have been considered as a deep and fascinating subject. They provide a natural connection between the evolution of a physical system with degenerate structure and differential geometry. Here we shall present a model where these concepts can be explicitly applied for quantum computation [Zan].

The physical setup consists of an energy degenerate quantum system on which we perform an adiabatic isospectral evolution described by closed paths in the parametric space of external variables. The corresponding evolution operators acting on the code-state in the degenerate eigenspace are given in terms of holonomies and we can use them as quantum logical gates. This is a generalization of the Berry phase or geometrical phase, to the non-abelian case, where a non-abelian adiabatic connection, $A$, is produced from the geometrical structure of the degenerate spaces. In particular, on each point of the manifold of the external parameters there is a code-state attached and a transformation between these bundles of codes is dictated by the connection $A$.

In order to apply this theoretical construction to a concrete example we employ a model with $\mathbf{C P}^{2}$ geometry, that is a complex projective manifold with two complex coordinates. This is interpreted as a qubit $[\mathbf{P a d}$. A further generalization with the tensor product of $m \mathbf{C P}^{2}$ models and additional interaction terms parametrized by the Grassmannian manifold, $\mathbf{G}(4,2)$, is interpreted as a model of quantum computer.

The initial code-state is written on the degenerate eigenspace of the system. The geometrical evolution operator is a unitary acting on it and it is interpreted as a logical gate. Due to adiabaticity the geometrical part of the evolution operator

1991 Mathematics Subject Classification. Primary 03.67.Lx, 03.65.Bz; Secondary 42.50.Dv.

The author was supported in part by TMR Network under the contract no. ERBFMRXCT96 $-0087$.

(c) 0000 (copyright holder) 
has a dimensionality equal to the degree of degeneracy of the eigenspace. Specific logical gates given by holonomies are constructed for a system with a tensor product structure resulting in universality while at the end a quantum optical application is sketched.

\section{Coset Space Geometry}

A transformation $U(n)$ between the states $|\alpha\rangle, \alpha=1, \ldots, n$ can be realized by all possible sub- $U(2)$ transformations between any two of those states, $|i\rangle$ and $|j\rangle$. A coset space can be produced as the factor with respect to some particular $U(2)$ symmetries of these transformations.

Examples of such constructions are given in the following:

- the $\mathbf{C P}^{2}$ projective space:

$$
\mathbf{C P}^{2} \cong \frac{U(3)}{U(2) \times U(1)}
$$

$|1\rangle$

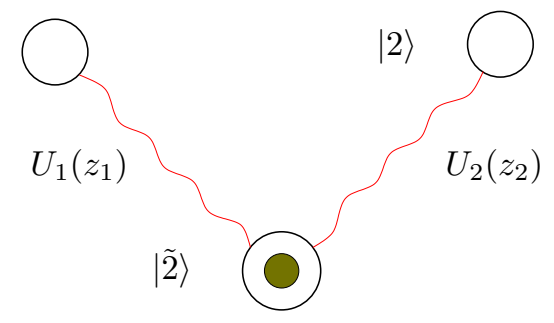

The lines denote $U(2)$ transformations between the states represented here by "holes". The $U(3)$ group could be interpreted by three lines connecting all the holes together. The distinction between "filled" and "unfilled" holes is due to the coset structure, which factors out the symmetry transformations, between $|1\rangle$ and $|2\rangle$, and denotes explicitly the non-symmetric ones between $|1\rangle$ or $|2\rangle$ and $|\tilde{2}\rangle$.

- the $\left(\mathbf{C P}^{2}\right)^{\times m} \times\left(\mathbf{G}(4,2)_{i n t}\right)^{\times(m-1)}$ product space:

$$
\cdots \frac{U(3)}{U(2) \times U(1)} \times \frac{U(3)}{U(2) \times U(1)},\left.\frac{U(4)}{U(2) \times U(2)}\right|_{i n t} \ldots
$$

$|1\rangle$

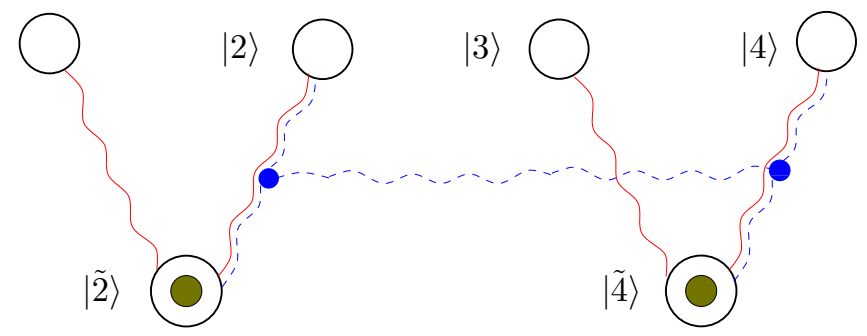

Transformations can be performed between the states $\{|1\rangle,|2\rangle\}$ and $\{|3\rangle,|4\rangle\}$ due to their connections with the states $\{|\tilde{2}\rangle,|\tilde{4}\rangle\}$, while an interaction in the tensor product space between $|24\rangle$ and $|\tilde{2} \tilde{4}\rangle$ gives transformations between those two sets. 
The transformations between only two states may be performed by linear operations with respect to $U(2)$ generators, while the combined transformations between the two qubits can be produced by bilinear generators which act simultaneously on the states of both of the $\mathbf{C P}^{2}$ models. The latter is denoted in the previous figure by the dashed lines, where the connection between the black dots indicates the simultanious action.

\section{Degeneracy, Adiabaticity and Holonomies}

Let us introduce the degenerate Hamiltonians $H_{0}^{1}$ and $H_{0}^{m}$ as follows

$$
H_{0}^{1}=\left[\begin{array}{lll}
0 & 0 & 0 \\
0 & 0 & 0 \\
0 & 0 & 1
\end{array}\right], \quad H_{0}^{m}=\sum_{m} H_{0}^{1} .
$$

The orbit, that is the parametric manifold of the unitary transformations which preserves the degenerate spectrum of $H_{0}^{1}$ is given by $\mathbf{C P}^{2}$. A sub-manifold of the orbit of $H_{0}^{m}$ in which we are interested in is given by the $\left(\mathbf{C P}^{2}\right)^{\times m} \times\left(\mathbf{G}(4,2)_{i n t}\right)^{\times(m-1)}$ product manifold.

A general transformation parametrized by the $\mathbf{C P}^{2}$ space is given by $\mathbf{U}(\mathbf{z}):=$ $U_{1}\left(z_{1}\right) U_{2}\left(z_{2}\right)$, with $U_{\alpha}\left(z_{\alpha}\right)=\exp G_{\alpha}\left(z_{\alpha}\right)=\exp \left(z_{\alpha}|\alpha\rangle\left\langle\tilde{2}\left|-\bar{z}_{\alpha}\right| \tilde{2}\right\rangle\langle\alpha|\right)$. The complex parameter $z_{\alpha}$ may be decomposed as $z_{\alpha}=\theta_{\alpha} \exp i \phi_{\alpha}$. Due to the $2 \times 2$ sub-form of $G_{\alpha}\left(z_{\alpha}\right)$ we can rewrite $U_{\alpha}\left(z_{\alpha}\right)$ as

$$
U_{\alpha}\left(z_{\alpha}\right)=\mathbf{1}_{\alpha}^{\perp}+\cos \theta_{\alpha} \mathbf{1}_{\alpha}+\frac{\sin \theta_{\alpha}}{\theta_{\alpha}} G_{\alpha}\left(z_{\alpha}\right),
$$

where $\mathbf{1}_{\alpha}^{\perp}=\mathbf{1}-\mathbf{1}_{\alpha}$ and $\mathbf{1}_{\alpha}=|\alpha\rangle\langle\alpha|+| \tilde{2}\rangle\langle\tilde{2}|$. For the Grassmannian manifold $\mathbf{G}(4,2)$ we have, for example, the $U(2)$ rotation in the tensor product basis of two qubits, between the states $|24\rangle$ and $|\tilde{2} \tilde{4}\rangle$, given by $\mathbf{U}(z)=\exp (z|24\rangle\langle\tilde{2} \tilde{4}|-\bar{z}| \tilde{2} \tilde{4}\rangle\langle 24|)$, with $z=\theta \exp i \phi$. The coordinates $\left\{\lambda^{a}\right\}=\{\boldsymbol{\theta}, \boldsymbol{\phi}\}$ provide the parametric space which the experimenters control.

In the four dimensional manifold $\mathbf{C P}^{2}$ with coordinates $\left\{\lambda^{a}\right\}$ a closed path, $C$, is drawn on a two-submanifold. Consider this evolution to be adiabatic as well as isospectral which is provided by the formula $H(\lambda(t))=\mathbf{U}(\lambda(t)) H_{0} \mathbf{U}^{\dagger}(\lambda(t))$. As a result the state of the system, $|\psi(t)\rangle$, stays on the same eigenvalue of the Hamiltonian, taken in our example to be $E_{0}=0$, without level-crossing.

At the end of the loop $C$, spanned in time $T=N \Delta t$, when divided in $N$ equal time intervals, we obtain

$$
\begin{aligned}
|\psi(T)\rangle \quad & =\mathbf{T} e^{-i \int_{0}^{T} \mathbf{U} H_{0} \mathbf{U}^{\dagger} d t}|\psi(0)\rangle \\
& =\mathbf{T} \lim _{N \rightarrow \infty} \prod_{i=1}^{N} \mathbf{U}_{i} e^{-i H_{0} \Delta t} \mathbf{U}_{i}^{\dagger}|\psi(0)\rangle \\
& =\mathbf{P} \lim _{N \rightarrow \infty}\left(\mathbf{1}+\sum_{i=1}^{N} A_{i} \Delta \lambda_{i}\right) \quad|\psi(0)\rangle \\
\text { with } \quad & A_{i}=\mathbf{U}_{i}^{\dagger} \frac{\Delta \mathbf{U}_{i}}{\Delta \lambda_{i}} \text { and } \quad \mathbf{U}_{i}=\mathbf{U}\left(\lambda\left(t_{i}\right)\right) .
\end{aligned}
$$


Hence, the state $|\psi(0)\rangle$ acquires a geometrical unitary operator given by the holonomy of a connection $A$ as

$$
\Gamma_{A}(C):=\mathbf{P} \exp \oint_{C} A, \quad \text { where } \quad\left(A^{\lambda^{a}}\right)_{\alpha \beta}:=\left\langle\alpha\left|\mathbf{U}^{\dagger}(\lambda) \frac{\partial}{\partial \lambda^{a}} \mathbf{U}(\lambda)\right| \beta\right\rangle .
$$

The states $|\alpha\rangle$ and $|\beta\rangle$ belong to the same degenerate eigenspace of $H_{0}$ and $\lambda^{a}$ 's are the real control parameters.

The produced unitary operator is an effect of the non-commutativity of the control transformations which produce effectively a curvature. In the case of the Berry phase produced for example in front of the spin states of an electron when placed in a magnetic field, the non-commutativity is between the $U(2)$ control unitaries which change the direction of the magnetic field in the three dimensional space. What is presented here is the generalization of the Berry phase to the nonabelian case.

The $\Gamma_{A}(C)$ 's produced by $\mathbf{C P}^{2}$ for various loops $C$, generate the whole $U(2)$,

$$
\left\{\Gamma_{A}(C) ; \forall C \in \mathbf{C P}^{2}\right\} \approx U(2) .
$$

In the case of $m$ qubits with their proper interactions, the produced group is $U\left(2^{m}\right)$,

$$
\left\{\Gamma_{A}(C) ; \forall C \in\left(\mathbf{C P}^{2}\right)^{\times m} \times\left(\mathbf{G}(4,2)_{i n t}\right)^{\times(m-1)}\right\} \approx U\left(2^{m}\right) .
$$

\section{Quantum Computation}

In order to perform quantum computation by using the above constructions we consider the following identifications:

$$
\begin{gathered}
\text { QUANTUM CODE } \equiv \text { Degenerate States, }|\psi(0)\rangle \\
\text { LOGICAL GATES } \equiv \text { Holonomies, } \Gamma_{A}(C)
\end{gathered}
$$

Let us first investigate the $\mathbf{C P}^{2}$ case. The basic question is how we can generate a general $U(2)$ element by moving along a closed path, $C$. Or in other words, for a specific $U \in U(2)$ which loop $C$ is such that $\Gamma_{A}(C)=U$. In general, $\forall \mathbf{g} \in$ $u(2) \exists$ loop $C \in \mathbf{C P}^{2}$ manifold, such that $\Gamma_{A}(C)=\exp \mathbf{g}$, which is the statement of irreducibility of the connection $A[\mathbf{Z a n}$. To answer the above question we perform the following analysis. The loop integral

$$
\oint_{C} A=\oint_{C} A_{\lambda^{1}} d \lambda^{1}+A_{\lambda^{2}} d \lambda^{2}+\cdots
$$

is the main ingredient of the holonomy. Due to the path ordering symbol it is not possible to just calculate it and evaluate its exponential, as in general the connection components do not commute with each other. Still it is possible to consider the following restrictions in the position of the loop. Choose $C$ such that:

- it belongs to one plane $\left(\lambda^{i}, \lambda^{j}\right)=\left(\theta_{i}, \phi_{j}\right)$ or $\left(\theta_{i}, \theta_{j}\right)$, hence only two components of $A$ are involved,

- the position of the plane is such that the connection, $A$, restricted on it become, $\left.A\right|_{\left(\lambda^{i}, \lambda^{j}\right)}=\left(A^{\lambda^{i}}=0, A^{\lambda^{j}}\right)$, that is these two components commute with each other. Still it is important that their related field strength component, $F_{i j}=\partial_{i} A_{j}-\partial_{j} A_{i}+\left[A_{i}, A_{j}\right]$, is non-vanishing in order to obtain a non-trivial holonomy. Such a requirement is possible for the $\mathbf{C P}^{2}$ model and for a wide class of other models. 
On the planes where those conditions are satisfied the evaluation of the holonomy is trivially given by just exponentiating the loop integral of the connection without worrying about the path ordering symbol. Hence,

$$
\Gamma_{A}(C)=\mathbf{P} \exp \oint_{C} A=\exp (\Sigma \mathbf{g})=\mathbf{1}_{2 \times 2} \cos \Sigma+\mathbf{g} \sin \Sigma
$$

where $\Sigma$ represents the area enclosed by the loop $C$ projected on the sphere associated with the compactified $\mathbf{C P}^{2}$ manifold. This area may be varied desirably. Furthermore, we are able to obtain a complete set of generators $\mathbf{g}$ by choosing $C$ to lie on different planes. In detail we may obtain for $\mathbf{g}$ the following forms

$$
\begin{aligned}
& -i|\alpha\rangle\langle\alpha| \quad:=-i \sigma_{\alpha}^{3}, \alpha=1,2 \\
& -i(-i|1\rangle\langle 2|+i| 2\rangle\langle 1|) \quad:=-i \sigma^{2}, \\
& -i(|1\rangle\langle 2|+| 2\rangle\langle 1|) \quad:=-i \sigma^{1} .
\end{aligned}
$$

The $\sigma_{\alpha}^{3}$ generators are similar to a Berry phase and they are produced by paths $C_{1}$ on the $\left(\theta_{\alpha}, \phi_{\alpha}\right)$ planes. The corresponding holonomy is the exponential of this generator multiplied by the area, $\Sigma_{1}$, of the surface the path $C_{1}$ encloses when projected on a sphere $S^{2}\left(2 \theta_{\alpha}, \phi_{\alpha}\right)$ with spherical coordinates $2 \theta_{\alpha}$ and $\phi_{\alpha}$,

$$
-i \sigma_{\alpha}^{3}: C_{1} \in\left(\theta_{\alpha}, \phi_{\alpha}\right) \rightarrow \Gamma_{A}\left(C_{1}\right)=\exp -i \Sigma_{1} \sigma_{\alpha}^{3} .
$$

The $\sigma^{2}$ generator is produced by a path $C_{2}$ along the plane $\left(\theta_{1}, \theta_{2}\right)$ positioned at $\phi_{1}=\phi_{2}=0$, while $\sigma^{1}$ is produced by a path, $C_{3}$, along a parallel plane positioned at $\phi_{1}=\frac{\pi}{2}$ and $\phi_{2}=0$. Their corresponding areas are $\Sigma_{2}$ and $\Sigma_{3}$. For example

$$
-i \sigma^{2}:\left.C_{2} \in\left(\theta_{1}, \theta_{2}\right)\right|_{\phi_{1}=0, \phi_{2}=0} \rightarrow \Gamma_{A}\left(C_{2}\right)=\exp -i \Sigma_{2} \sigma^{2} .
$$

Altogether we have $2^{2}$ independent generators spanning the Lie algebra of $U(2)$.

For the case of the two qubit interaction the corresponding connection components are given by

$$
A_{\theta}=\operatorname{diag}(0,0,0,0), A_{\phi}=\operatorname{diag}\left(0,0,0,-i \sin ^{2} \theta\right)
$$

which are written in the basis $\{|13\rangle,|14\rangle,|23\rangle,|24\rangle\}$. A loop $C$ on the $(\theta, \phi)$ plane will produce the following holonomy

$$
\Gamma_{A}(C)=\operatorname{diag}\left(1,1,1, e^{-i \Sigma}\right), \quad \Sigma=\int_{D(C)} d \theta d \phi \sin 2 \theta,
$$

where $\Sigma$ can also be interpreted as an area on the sphere $S^{2}(2 \theta, \phi)$.

\section{One and Two Qubit Logical Gates}

By performing appropriate loops we can obtain one qubit phase rotations as well as two qubit gates such as a controlled phase rotation $U_{C P H}$.

Analytically, by spanning the indicated areas we may obtain

$$
U_{1}=\exp \left[\begin{array}{cc}
-i \Sigma_{1} & 0 \\
0 & 0
\end{array}\right], \quad U_{2}=\exp \left[\begin{array}{cc}
0 & 0 \\
0 & i \Sigma_{1}
\end{array}\right], \quad U_{3}=\exp \left[\begin{array}{cc}
0 & -\Sigma_{2} \\
\Sigma_{2} & 0
\end{array}\right] \text {. }
$$

The combinations

$$
U_{1} U_{2}=\exp \left(-i \sigma_{3} \Sigma_{1}\right), U_{3}=\exp \left(-i \sigma_{2} \Sigma_{2}\right)
$$

can give any $U(2)$ transformation and hence any one qubit rotation.

For the two qubit gates we can construct easily the controlled rotation $U_{C P H}=$ $\operatorname{diag}(1,1,1, \exp -i \Sigma)$ between any pair of qubits. It is generated by a loop $C$ on the 
$(\theta, \phi)$ plane. Together with the one qubit rotations they provide a universal set of gates.

\section{Epilogue}

Apart from the intriguing theoretical formulation of holonomic computation there are several aspects of it, which have appealing technical advantages. Without overlooking the difficulties posed to an experimenter for performing continuous control over a system in order to span a loop, there are several unique characteristics of it, which await for exploitation. For example, robustness of the control procedure in terms of the spanned area, according to errors in the actual form of the performed loop, as well as the isolation of the degenerate states as a calculational space may prove to be advantages worth exploring.

In quantum optics displacing devices, squeezing devices and interferometers acting on laser beams can provide the control parameters for the holonomic computation. Each laser beam is placed in a non-linear Kerr medium with degenerate Hamiltonian $H_{0}=n(n-1)$, where $n$ is the photon numbering operator. The degenerate states $|0\rangle$ and $|1\rangle$ are the basis for encoding one qubit which is manipulated by displacing and squeezing devices. Any two qubit interactions can be implemented by interferometers [Cho].

It is challenging for the experimenters to produced the desired closed paths.

\section{References}

[Sha] Geometric Phases in Physics, A. Shapere and F. Wilczek, Eds. World Scientific (1989).

[Wil] F. Wilczek and A. Zee., Phys. Rev. Lett. 52, 2111 (1984).

[Zan] P. Zanardi and M. Rasetti, Phys. Lett. A 264, 94 (1999), quant-ph/9904011.

[Pac] J. Pachos, P. Zanardi and M. Rasetti, Phys. Rev. A 61 010305(R), quant-ph/9907103.

[Cho] J. Pachos and S. Chountasis, quant-ph/9912093.

Institute for Scientific Interchange Foundation, Villa Gualino, Viale Settimio Severo 65, I-10133 Torino, Italy

E-mail address: pachos@isiosf.isi.it 